\title{
Magic Numbers of the Great Pyramid: A Surprising Result
}

\author{
Hans Hermann Otto \\ Department of Materials Science and Crystallography, Clausthal University of Technology, Clausthal-Zellerfeld, Germany \\ Email: hhermann.otto@web.de
}

How to cite this paper: Otto, H.H. (2020) Magic Numbers of the Great Pyramid: A Surprising Result. Journal of Applied Mathematics and Physics, 8, 2063-2071. https://doi.org/10.4236/jamp.2020.810154

Received: August 24, 2020

Accepted: October 7, 2020

Published: October 10, 2020

Copyright $\odot 2020$ by author(s) and Scientific Research Publishing Inc. This work is licensed under the Creative Commons Attribution International License (CC BY 4.0).

http://creativecommons.org/licenses/by/4.0/

\begin{abstract}
Recently attention has been drawn to the frequently observed fifth power of the golden mean in many disciplines of science and technology. Whereas in a forthcoming contribution the focus will be directed towards Fibonacci number-based helical structures of living as well as inorganic matter, in this short letter the geometry of the Great Pyramid of the ancient Egyptians was investigated once more. The surprising main result is that the ratio of the in-sphere volume of the pyramid and the pyramid volume itself is given by $\pi \cdot \varphi^{5}$, where $\varphi=0.618033987 \cdots$ is nature's most important number, the golden mean. In this way not only phase transitions from microscopic to cosmic scale are connected with $\varphi^{5}$, also ingenious ancient builders have intuitively guessed its magic before.
\end{abstract}

\section{Keywords}

Geometry, Great Pyramid, In-Sphere Volume, Golden Mean, Fifth Power of Golden Mean, Fibonacci Number 13, Curious Angel Arcsin $\left(\varphi^{5}\right)$

\section{Introduction}

Recently, attention has been drawn to the more than expected occurrence of the fifth power of the golden mean in different disciplines of science and technology [1]. The golden mean or golden ratio is an omnipresent number in nature, found in the architecture of living creatures as well as human buildings, music, finance, medicine, philosophy, and of course in physics and mathematics including quantum computation [2] [3] [4] [5]. It is the most irrational number known and a number-theoretical chameleon with a self-similarity property. When writing down its continued fraction representation, which is the simples of all (see Equation (1)), one can catch an idea of why nature works so effectively, so that Olson et al. [4] arrive at the statement that the whole universe functions as a golden 
supercomputer.

In this contribution we use the golden mean $\varphi$ as defined in Equation (1) besides big $\phi=1+\varphi=\varphi^{-1}$. The reader can found compacted information about infinitely continued fraction representations in reference [6] [7]. The golden mean $\varphi$ and its fifth power are represented by

$$
\begin{gathered}
\varphi=\frac{\sqrt{5}-1}{2}=\frac{1}{1+\frac{1}{1+\frac{1}{1+\cdots}}}=0.6180339887 \cdots \\
\varphi^{5}=\frac{1}{11+\frac{1}{11+\frac{1}{11+\cdots}}}=0.0901699 \cdots
\end{gathered}
$$

The seminal $E$-infinity $\left(\mathcal{\varepsilon}^{\infty}\right)$ approach developed by El Naschie years ago and applied mainly to cosmological questions based on this simple principle of nature, too [8] [9] [10]. It originates from a fractal Cantorian set theory [11] as a number-theoretical route of physics for explaining the dualism between particles and waves that can help solving cosmological mysteries such as dark matter and dark energy [12]. A probabilistic quantum entanglement calculation delivers effective quantum gravity formulas for the cosmological mass (energy) constituents. The baryonic matter amount $e_{M}$ resulted according to El Naschie and scientific fellows in

$$
e_{M}=\frac{\varphi^{5}}{2}=0.04508497
$$

This result points towards the significance of the fifth power of the golden mean. Obviously this number is fundamental and was reported before in the theory of phase transitions (see Chapter 2). Furthermore, the fifth power of the golden mean could be identified with the maximum of Hardy's quantum probability of two particles [13] [14], and later it is found again in Suleiman's scale-free Information Relativity Theory (IRT) as maximum of the matter energy density $\left(e_{M}\right)_{\max }$ indicating a phase transition of cosmic scale [15]

$$
\left(e_{M}\right)_{\max }=e_{o} \frac{1-\varphi}{1+\varphi} \varphi^{2}=e_{o} \varphi^{5}=e_{o} \cdot 0.09016994 \cdots
$$

Seeking for a more geometry-based interpretation of the fifth power of the golden mean, the present author has found a surprising result that will be formulated in the next chapters revealing more secrets of the Great Pyramid of Giza. The numerous contributions about the mysterious geometry of this pyramid can't be quoted here. They would only contribute marginally to the subject. However, reference is recommended again to the work initiated by Olson [2] [3] [4]. A short summary about the appearance of the fifth power of the golden mean is given in Chapter 2. Turning then to Chapter 3, the volume of a special quadratic pyramid (Great Pyramid) is compared to that of its in-sphere volume. These considerations are supplemented by a further Chapter 4 about the out- 
standing importance of Fibonacci number 13, again with reference to the Great Pyramid.

\section{Appearance of the Fifth Power of the Golden Mean}

In the following overview scientific fields were summarized according to [1], where the fifth power of the golden mean is involved indicating that this number is frequently observed all around the world:

1) Phase transition of the hard hexagon lattice gas model [16] [17];

2) Phase transition of the hard square lattice gas model [17] [18];

3) One-dimensional hard-boson model [19];

4) Curious angle $\arcsin \left(\varphi^{5}\right)$ [1];

5) Baryonic matter relation according to the $E$-infinity theory [12];

6) Maximum quantum probability of two particles [13] [14];

7) Maximum of matter energy density [15];

9) Reciprocity relation between matter and dark matter [20] [21];

9) Superconductivity phase transition [22] [23];

10) Geometry of the Great Pyramid (this publication).

Topic 10 of this general overview will now be considered in detail in the following chapters pointing to a not expected beautiful result. Whether also the fifth power of the golden mean, apart from the golden mean, can be identified in the DNA genetic code is under discussion.

\section{Relation between the Volume of the Great Pyramid and Its In-Sphere Volume}

We were intuitively interested in the relation between the volume of the Great Pyramid of Giza and its in-sphere volume. Whereas in case of a general tetrahedron, for instance, a contiguous derivation of radius and center of the in-sphere was not solved as yet, very recently such derivation was delivered from P. P. Klein of the TU Clausthal [24]. Contrary, in case of a quadratic pyramid the in-sphere can be quite simply derived from the given data explained in Figure 1, in which a mid-plane triangle cut down the apex of the pyramid is displayed together with the projected in-sphere in yellow color.

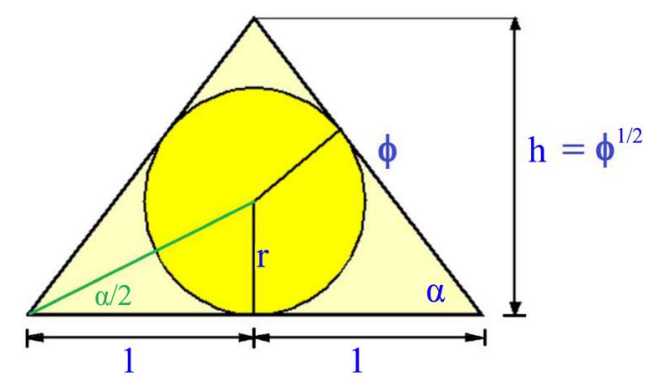

Figure 1. Cut through the middle of a Great Pyramid's face down the apex with yellow displayed in-sphere projection. Big $\phi$ denotes the inverse of $\varphi: \quad \phi=\varphi^{-1}=1+\varphi$. 
Half the base edge is normed to unity, $a / 2=1$, and the side legs have the numerical value of $\phi=\varphi^{-1}$. Then the height $h$ of the pyramid is

$$
h=\sqrt{\phi}=\varphi^{-1 / 2}=1.2720196 \cdots
$$

According to Figure 1 the outlined angle on the base is

$$
\alpha=\arccos (\varphi)=51.82729 \text {. }
$$

One can further derive that the radius of the in-sphere yields

$$
r=\tan \left(\frac{\alpha}{2}\right)=0.485868 \cdots=\varphi^{3 / 2}
$$

Now we want to compare the volume of the pyramid with the in-sphere volume. The volume of the quadratic pyramid with full base $a$ and height $h$ is given by

$$
V_{p y r}=\frac{1}{3} a^{2} h=\frac{4}{3} \varphi^{-1 / 2}
$$

and the volume of the in-sphere is

$$
V_{s p h}=\frac{4}{3} \pi r^{3}=\frac{4}{3} \pi \varphi^{9 / 2}
$$

Finally, the volume ratio gives the astonishing result apparently not widely known as yet

$$
\frac{V_{s p h}}{V_{p y r}}=\pi \cdot \varphi^{5}
$$

One is again faced with the fifth power of the golden mean. However, it would be purely speculative to connect the maximum of the matter energy density of the cosmos with such secret of the Great Pyramid [12] [15]. It seems as if the sun is inside the pyramid. I am happy contributing this result especially to my Egyptian friends and the Egyptian community.

In connection with the Great Pyramid, mention may be made of the vision of the great inventor Nicola Tesla to harvest free energy from what we now characterize as dark components of matter respectively energy. The reader can find more information about this discussion in the references [15] and [25].

If one approximates $\pi$ by a $\varphi$-based relation according to [26] the result is expressed solely by $\varphi$ giving

$$
\pi \cdot \varphi^{5}=0.283277 \cdots \approx \frac{6}{5} \varphi^{3}=0.283281 \cdots
$$

Another approximation is curious regarding the angle of $\alpha / 2$ (see Equation (4) and Figure 1) in connection with the angle $\beta=15.5224^{\circ}$ given in [1] [27]

$$
\frac{\alpha}{2}=\arctan (r)=\arctan \left(\varphi^{\frac{3}{2}}\right) \approx 5 \cdot \arcsin \left(\varphi^{5}\right) \approx \frac{5}{3} \beta
$$

In addition, the surface ratio between the pyramid and its in-sphere is calculated. The result would better fit into the next chapter but is given in Appendix. 
In Appendix also another geometry-based approximation of the fifth power of the golden mean is derived for comparison.

\section{The Fibonacci Number 13}

Among the numbers of the Fibonacci series [28] [29], the numbers 5 as well as 13 seem to be most important. Whereas number 5 is involved in the definition of the golden mean, number 13 is found as helix repetition number for instance in tubulin protein, thought to be the location from where our thinking and consciousness originates [1] [4] [30] [31]. This number is very special because its square root can be approximated by $2+\varphi^{-1}$

$$
\sqrt{13}=3.60555 \cdots \approx 2+\varphi^{-1}=3.6180339887 \cdots
$$

Furthermore, the exact adjustment connects the number 13 to the fifth power of $\varphi$

$$
2+\varphi^{-1}=\sqrt{5} \varphi^{-1}=\sqrt{13+\varphi^{5}}=\sqrt{13.0901699 \cdots}=3.6180339887 \cdots
$$

In addition it yields

$$
2+\varphi^{-1}=\sqrt{13+\varphi^{5}}=\sqrt{2+\varphi^{-5}}
$$

Remarkably, one can connect $\sqrt{13+\varphi^{5}}$ to the geometry of the Great Pyramid of Giza. Denoting the edge of the pyramid (from apex to base) as $s$, one can determine its relative length to be

$$
s=\sqrt{\varphi^{-2}+1}=\sqrt{\varphi^{-1}+2}=1.90211303 \cdots
$$

By using equation (14) the second power of $s$ gets

$$
s^{2}=\varphi^{-1}+2=\sqrt{13+\varphi^{5}}=\sqrt{2+\varphi^{-5}}
$$

and further

$$
\varphi^{5}=s^{4}-13=\left(s^{4}-2\right)^{-1}
$$

This is another way of interpreting the beauty geometry of the Great Pyramid using the fifth power of the golden mean besides Fibonacci number 13. A sketch of the pyramid is illustrated in Figure 2.

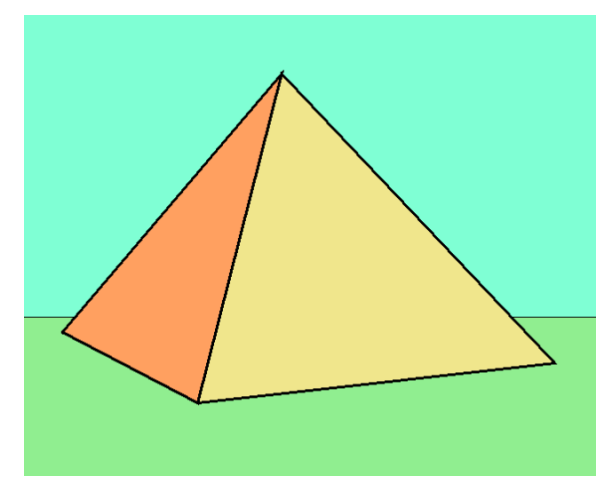

Figure 2. Artistic sketch of the Great Pyramid designed by the author. 
The high of the pyramid was originally $h=146.59 \mathrm{~m}$ (280 cubits) and is now about $138.75 \mathrm{~m}$. Its base is about $a=230.36 \mathrm{~m}$ (440 cubits). The ratio yields $h / a=0.636363 \cdots$ and can be approximated by $\frac{\sqrt{\phi}}{2}=0.6360098 \cdots$ (see Figure 1).

\section{Conclusion}

This contribution may be of general interest because it connects results of different disciplines of science and technology with the beauty of the mathematical knowledge of the ancient Egyptian builders of the pyramids. The present author has recently shown that besides the golden mean its fifth power $\varphi^{5}$ is also most important in describing different problems in science and technology such as phase transformations of microscopic to cosmic scale. Nevertheless, it was a surprise to elicit the Great Pyramid of Giza another secret by geometrical investigation of its golden ratio architecture. The ratio of the in-sphere volume of the pyramid to that of the pyramid itself turned out to be beautiful simply yielding $\pi \cdot \varphi^{5}$. It is a further step towards understanding our world and states once more that the golden ratio is the great numerical dominator of our world leaving also a fingerprint in the DNA genetic code [32]. It is under discussion whether also the fifth power of the golden mean can be identified in the genetic code [33].

\section{Conflicts of Interest}

The author declares no conflicts of interest regarding the publication of this paper.

\section{References}

[1] Otto, H.H. (2020) Phase Transitions Governed by the Fifth Power of the Golden Mean and Beyond. World Journal of Condensed Matter Physics, 10, 135-158. https://doi.org/10.4236/wjcmp.2020.103009

[2] Olson, S. (2006) The Golden Section: Nature's Greatest Secret. Bloomsbury, London, $64 \mathrm{p}$.

[3] Olson, S. (2018) Reassessing the Roots of Theosophy: In Pursuit of the Precious Stone. Insight.

[4] Olsen, S., Marek-Crnjak, L., He, J.H. and El Naschie, M.S. (2020) A Grand Unification of the Sciences, Art \& Consciousness: Rediscovering the Pythagorean Plato's Golden Mean Number System. Journal of Progressive Research in Mathematics, 16, 2880-2931.

[5] Sherbon, M.A. (2014) Fundamental Nature of the Fine-Structure Constant. International Journal of Physical Research, 2, 1-9. https://doi.org/10.14419/ijpr.v2i1.1817

[6] Otto, H.H. (2017) Continued Fraction Representations of Universal Numbers and Approximations. Researchgate.net, 1-4.

[7] Andrews, G.E. (1981) Ramanujan's "Lost” Notebook. III. The Rogers-Ramanujan Continued Fraction. Advances in Mathematics, 41, 186-208. https://doi.org/10.1016/0001-8708(81)90015-3

[8] El Naschie, M.S. (2013) Quantum Entanglement: Where Dark Energy and Negative 
Gravity plus Accelerated Expansion of the Universe Comes from. Journal of Quantum Information Science, 3, 57-77. https://doi.org/10.4236/jqis.2013.32011

[9] El Naschie, M.S. (2018) From the Logical Foundation and the Derivation of E = $\left(\mathrm{mc}^{2} / 22\right)+\left(\mathrm{mc}^{2}\right)(21 / 22)=\mathrm{mc}^{2}$ to the Fractal Nature of Reality. International Journal of Applied Science and Mathematics, 5, 1-2.

[10] Marek-Crnjac, L. (2013) Cantorian Space-Time Theory. Lambert Academic Publishing, Saarbrücken, 1-50.

[11] Cantor, G. (1932) Gesammelte Abhandlungen mathematischen und philosophischen Inhalts. Springer, Berlin. https://doi.org/10.1007/978-3-662-00274-2

[12] El Naschie, M.S. (2017) Elements of a New Set Theory Based Quantum Mechanics with Application in High Quantum Physics and Cosmology. International Journal of High Energy Physics, 4, 65-74. https://doi.org/10.11648/j.ijhep.20170406.11

[13] Hardy, L. (1993) Nonlocality for Two Particles without Inequalities for Almost All Entangled States. Physical Review Letters, 71, 1665-1668.

https://doi.org/10.1103/PhysRevLett.71.1665

[14] Mermin, N.D. (1994) Quantum Mysteries Refined. American Journal of Physics, 62, 880-887. https://doi.org/10.1119/1.17733

[15] Suleiman, R. (2019) Relativizing Newton. Nova Scientific Publisher, New York, $1-207$.

[16] Baxter, R.J. (1980) Hard Hexagons: Exact Solution. Journal of Physics A, 13, L61-L70. https://doi.org/10.1088/0305-4470/13/3/007

[17] Baxter, R.J. (1982) Exactly Solved Models in Statistical Mechanics. Academic Press, London.

[18] Baxter, R.J., Enting, I.G. and Tsang, S.K. (1980) Hard-Square Lattice Gas. Journal of Statistical Physics, 22, 465-489. https://doi.org/10.1007/BF01012867

[19] Fendley, P., Sengupta, K. and Sanchev, S. (2004) Competing Density-Wave Orders in a One-Dimensional Hard-Boson Model. Physical Review B, 69, Article ID: 075106. https://doi.org/10.1103/PhysRevB.69.075106

[20] Otto, H.H. (2018) Reciprocity Relation between the Mass Constituents of the Universe and Hardy's Quantum Entanglement Probability. World Journal of Condensed Matter Physics, 8, 30-35. https://doi.org/10.4236/wjcmp.2018.82003

[21] Otto, H.H. (2020) Reciprocity as an Ever-Present Dual Property of Everything. Journal of Modern Physics, 11, 98-121. https://doi.org/10.4236/jmp.2020.111007

[22] Otto, H.H. (2016) A Different Approach to High- $\mathrm{T}_{c}$ Superconductivity: Indication of Filamentary-Chaotic Conductance and Possible Routes to Superconductivity above Room Temperature. World Journal of Condensed Matter Physics, 6, 244-260. https://doi.org/10.4236/wjcmp.2016.63023

[23] Otto, H.H. (2019) Super-Hydrides of Lanthanum and Yttrium: On Optimal Conditions for Achieving near Room Temperature Superconductivity. World Journal of Condensed Matter Physics, 9, 22-36. https://doi.org/10.4236/wjcmp.2019.91002

[24] Klein, P.P. (2020) The Insphere of a Tetrahedron. Applied Mathematics, 11, 601-612. https://doi.org/10.4236/am.2020.117041

[25] El Naschie, M.S. and He, J.-H. (2018) Tesla's Dream from a Modern Quantum Spacetime View Point. Nonlinear Science Letters A, 9, 36-43.

[26] Otto, H.H. (2017) Should We Pay More Attention to the Relationship between the Golden Mean and the Archimedes' Constant? Nonlinear Science Letters A, 8, 410-412.

[27] Fang, F., Irvin, K., Kovacs, J. and Sadler, G. (2013) Cabinet of Curiosities: The Interesting Geometry of the Angle $\beta$. 
[28] Pisano, L. (1202) Fibonacci's Liber Abaci (Book of Calculation). Biblioteca a Nazionale di Firenze.

[29] Lucas, E. (1891) Theorie des nombres. Gauthier-Villars, Paris.

[30] Penrose, R. and Hamedoff, S.R. (1995) Quantum Computing in Microtubules: Self-Collapse as a Possible Mechanism for Consciousness. Toward a Science of Consciousness. MIT Press, Cambridge.

[31] Penrose, R. and Hamedoff, S.R. (2011) Consciousness in the Universe: Neuroscience, Quantum Space-Time Geometry and Orch OR Theory. Journal of Cosmology, 14, 1-50.

[32] Planat, M., Aschheim, R.A., Amaral, M.M., Fang, F. and Irvin, K. (2020) Complete Quantum Information in the DNA Genetic Code. Researchgate.net, 1-14. https://doi.org/10.20944/preprints202007.0403.v1

[33] Otto, H.H. (2020) A Varied Quartic Polynomial Modeling the Double-Helix Genetic Code. Researchgate.net, Pre-Publication, 1-5. 


\section{Appendix}

\section{Surface ratio between pyramid and its in-sphere:}

The entire surface of the pyramid is composed of the outer surface and the base surface yielding

$$
O_{p y r}=4 \varphi^{-1}+4=4 \varphi^{-2}
$$

The surface of the in-sphere is

$$
O_{\text {sph }}=4 \pi r^{2}=4 \pi \varphi^{9 / 4}
$$

Then the surface ratio is calculated to be

$$
\frac{O_{s p h}}{O_{p y r}}=\pi \varphi^{13 / 4}
$$

In this way, the Fibonacci number 13 is once more found in a geometric derivation.

\section{Another Geometry-Based Approximation of $\varphi^{5}$ :}

Another simple relation leads to the following reliable result, applying the in-sphere radius for the hexagon $r_{i, h}=a \cdot \frac{\sqrt{3}}{2}$ and the out-sphere radius for a pentagon $r_{o, p}=\frac{a}{\sqrt{2-\varphi}}($ see Figure A1)

$$
5 \cdot\left(\frac{r_{i, h}}{r_{\text {o.p }}}-1\right)=0.0903696046 \cdots
$$

When enlarging the $r_{o, p}$ value by a factor of only $1.00003923 \ldots$ then the exact value of $\varphi^{5}$ can be confirmed. Extracting a value of $\varphi^{*}$ by recasting the relation (A4) using the approximation

$$
\left(\varphi^{*}\right)^{5} \approx 5 \varphi^{*}-3
$$

one yields

$$
\varphi^{*}=\frac{\sqrt{3}}{2} \sqrt{2-\varphi}-\frac{2}{5}=0.61807392 \cdots
$$

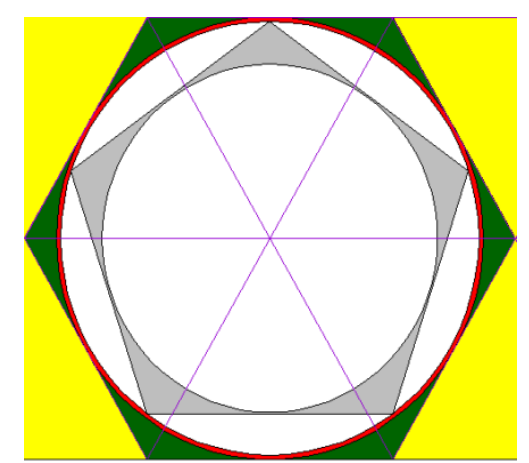

Figure A1. Hexagon (green) and pentagon (gray) displayed with a common edge length and center. The red outlined ring is limited by the radii $r_{i, h}$ (in-sphere radius of a hexagon) and $r_{o, p}$ (out-sphere radius of a pentagon). 